\title{
Les fractures du zygoma : à propos de 276 cas
}

\section{RÉSUMÉ}

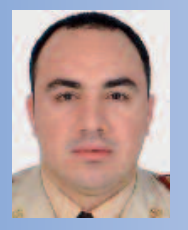

Lahcen KHALFI

Médecin résidant,

Service de chirurgie plastique, maxillo-faciale et stomatologie.

Hôpital militaire d'Instruction Mohammed V,

Rabat, Maroc.

\section{Abdeljalil ABOUCHADI}

Professeur assistant,

Service de chirurgie plastique, maxillo-faciale et stomatologie.

Hôpital militaire d'Instruction Mohammed V,

Rabat, Maroc.

\section{Jalal HAMAMA}

Médecin résidant,

Service de chirurgie plastique, maxillo-faciale et stomatologie.

Hôpital militaire d'Instruction Mohammed V, Rabat, Maroc.

\section{Karim ELKHATIB}

Professeur assistant,

Service de chirurgie plastique, maxillo-faciale et stomatologie.

Hôpital militaire d'Instruction Mohammed V

Rabat, Maroc.

\section{Mohamed NASSIH}

Professeur,

Service de chirurgie plastique, maxillo-faciale et stomatologie.

Hôpital militaire d'Instruction Mohammed V,

Rabat, Maroc.

\section{Abdelkader RZIN}

Professeur,

Service de chirurgie plastique, maxillo-faciale et stomatologie.

Hôpital militaire d'Instruction Mohammed V, Rabat, Maroc.

Introduction : les fractures de l'os zygomatique revêtent une multitude de variétés anatomo-cliniques à l'origine de retentissement fonctionnel et morphologique. Le but de ce travail est de déterminer et de préciser les circonstances de survenue, la sémiologie clinique et radiologique, l'attitude thérapeutique et le devenir à moyen terme de ces patients.

Matériel et méthodes : une étude rétrospective étant menée sur des patients ayant présenté une fracture isolée de l'os zygomatique traitée au service de chirurgie maxillo-faciale et chirurgie plastique de l'hôpital militaire d'instruction Mohammed V Rabat, entre mars 1999 et avril 2009.

Résultats : on a inclus 276 dossiers, l'âge moyen des patients est de 32 ans avec une nette prédominance masculine $(89,5 \%)$. Les étiologies ont été dominées par les accidents de la voie publique (64,2\%). Le diagnostic a été surtout clinique, confirmé par la radiologie. La réduction ouverte a été effectuée dans 173 cas tandis que 31,9\% des cas ont nécessité une réduction fermée. Les séquelles les plus fréquentes ont été représentées par les troubles de la sensibilité (19,2\%).

Discussion : l'évaluation préopératoire doit inclure une évaluation ophtalmologique complète. Les principes chirurgicaux impliqués dans la gestion des fractures du zygoma sont principalement liés à leur réduction. Cette expérience valide certaines indications pour la réduction orthopédique et souligne l'intérêt de la réduction sanglante avec fixation par mini-plaques vissées. L'indication chirurgicale dépend du type de fracture.

- fracture du zygoma

- Maroc 


\section{Introduction}

$>$

La fracture de l'os zygomatique est la deuxième fracture la plus fréquente en chirurgie maxillo-faciale.

Les séquelles fonctionnelles et morphologiques, souvent intriquées, sont ainsi fréquentes, ce qui incite à réaliser un bilan clinique et radiologique précis et rigoureux ainsi qu'une prise en charge chirurgicale adéquate et précoce qui n'est pas toujours facile.

À la lumière de cette étude, on a souhaité analyser notre stratégie diagnostique et thérapeutique afin d'aboutir à la conduite la plus satisfaisante en termes de résultat.

\section{Matériel et méthodes}

Notre étude est rétrospective et a porté sur 276 patients ayant présenté une fracture isolée de l'os zygomatique traités au service de chirurgie maxillo-faciale et chirurgie plastique de I'hôpital militaire d'instruction Mohammed V à Rabat, entre mars 1999 et avril 2009.

On n'a retenu que les fractures de l'os zygomatique avec ou sans extension vers l'os maxillaire. Les critères d'inclusion ont été l'obtention d'un recul minimal radiographique et clinique supérieur à un an.

Tous les patients ont bénéficié d'un examen clinique complet à la recherche de signes fonctionnels (diplopie, limitation de l'ouverture buccale, hypoesthésie sous-orbitaire) et morphologiques (affaissement de la pommette, énophtalmie). Un examen ophtalmologique spécialisé a été réalisé systématiquement, complété par un test de Lancaster à la demande.

La radio en incidence Blondeau a été demandée en première intention, complétée en cas de besoin d'une TDM.

La classification de Zingg (fig. 1) a été adoptée comme référence [1]. Nous avons adjoint une entité particulière qui est la fracture simple iso- lée du corps, et avons inclus ce type de fracture dans la catégorie $D$.

- Catégorie A : fracture isolée de l'un des trois processus de l'os zygomatique:

- le processus temporal A1 formant l'arcade zygomatique ;

- le processus frontal A2 formant le rebord orbitaire latéral ;

- le processus maxillaire A3 formant la margelle infra-orbitaire.

- Catégorie B : fracture-disjonction de l'os zygomatique avec rupture des quatre attaches (fig. 1).

- Catégorie C : fracture comminutive associant un fracas du zygoma aux lésions de la catégorie B (fig. 2).

- Catégorie $\mathbf{D}$ : fracture isolée du corps.

La prise en charge a dépendu du type de la fracture, allant de la réduction percutanée par crochet de Ginestet ou Dingman à la réduction sanglante avec ostéosynthèse. La margelle infra-orbitaire et le plancher de l'orbite ont été abordés à travers une incision sous-tarsale. La jonction fronto-zygomatique a été abordée par une incision latérale au niveau de la queue du 
sourcil. La voie vestibulaire supérieure a été utilisée pour aborder le cintre maxillo-zygoma- tique. Les fractures ont été stabilisées par des mini-plaques vissées en titane.
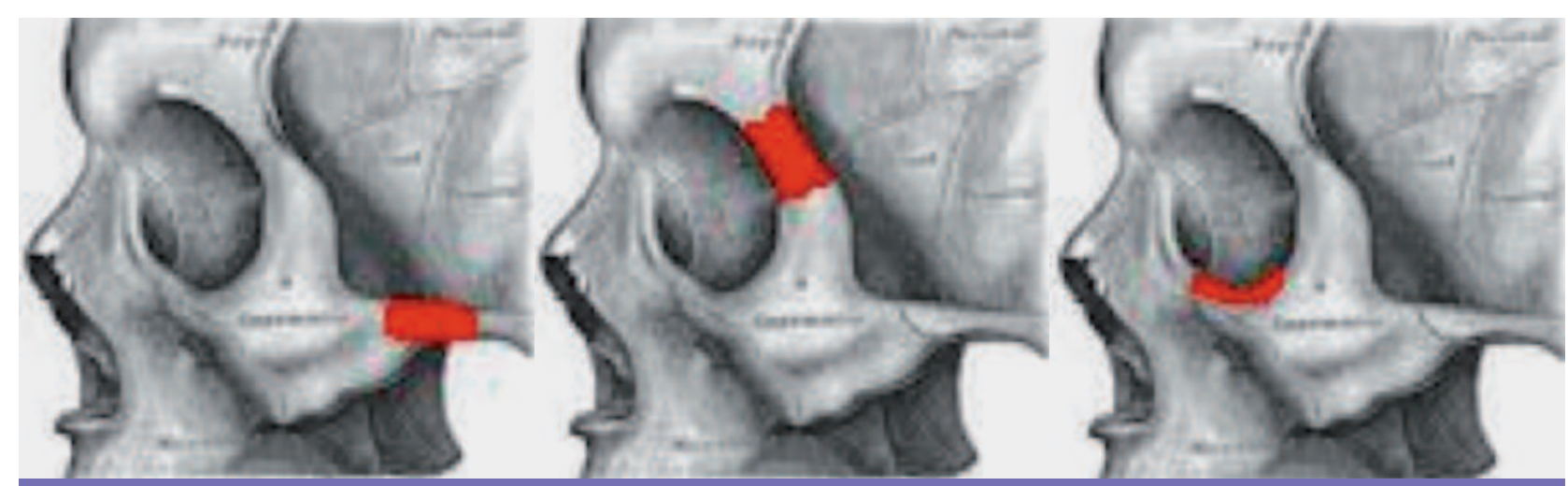

A1: fracture isolée de l'arcade zygomatique.

A2 : fracture isolée du rebord orbitaire latéral.
A3 : fracture isolée du rebord infraorbitaire.

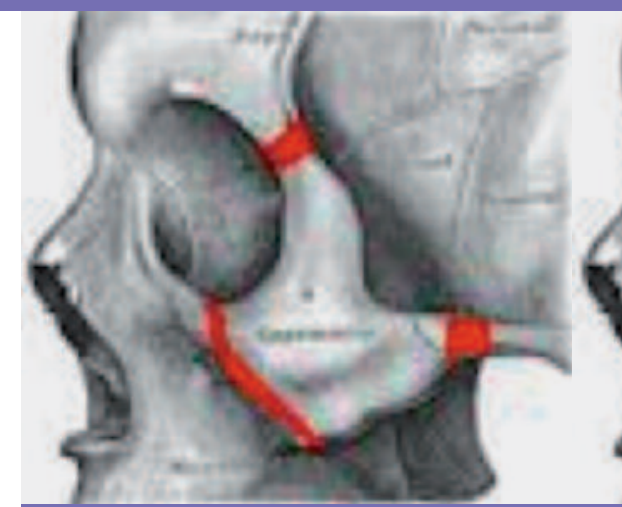

B : fracture disjonction.

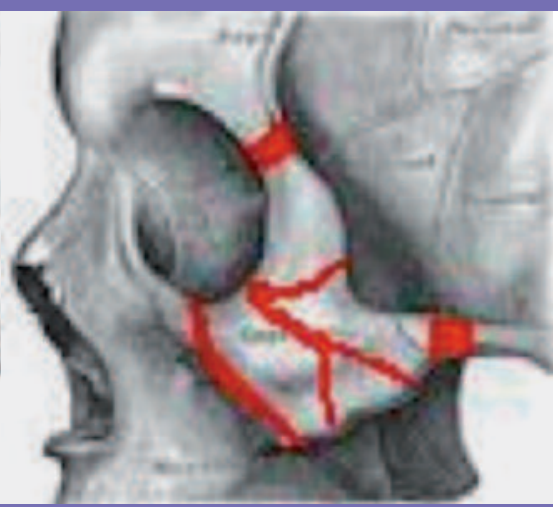

C : fracture comminutive.

Fig. 1 Classification de Zingg des fractures zygomatiques.

\section{Résultats}

Parmi les 1283 patients admis pour prise en charge d'un traumatisme maxillo-facial, 276 patients ont présenté une fracture isolée de l'os zygomatique soit $21,5 \%$.

L'âge moyen des patients est de 32 ans avec une nette prédominance masculine de l'ordre de $89,5 \%$.

Les accidents de la voie publique ont été la cause principale (177 cas), suivis des actes de violence (38 cas), des accidents de sport (33 cas) et des accidents domestiques (28 cas).

Le délai de la prise en charge a été inférieur à 48 heures dans $92 \%$ des cas.

La majorité des patients a présenté des troubles morphologiques (fig. 2), à savoir : œdème et ecchymose (252 cas), affaissement de la pommette (176 cas) (fig. 3). Une énophtalmie a été retrouvée chez 19 patients soit 


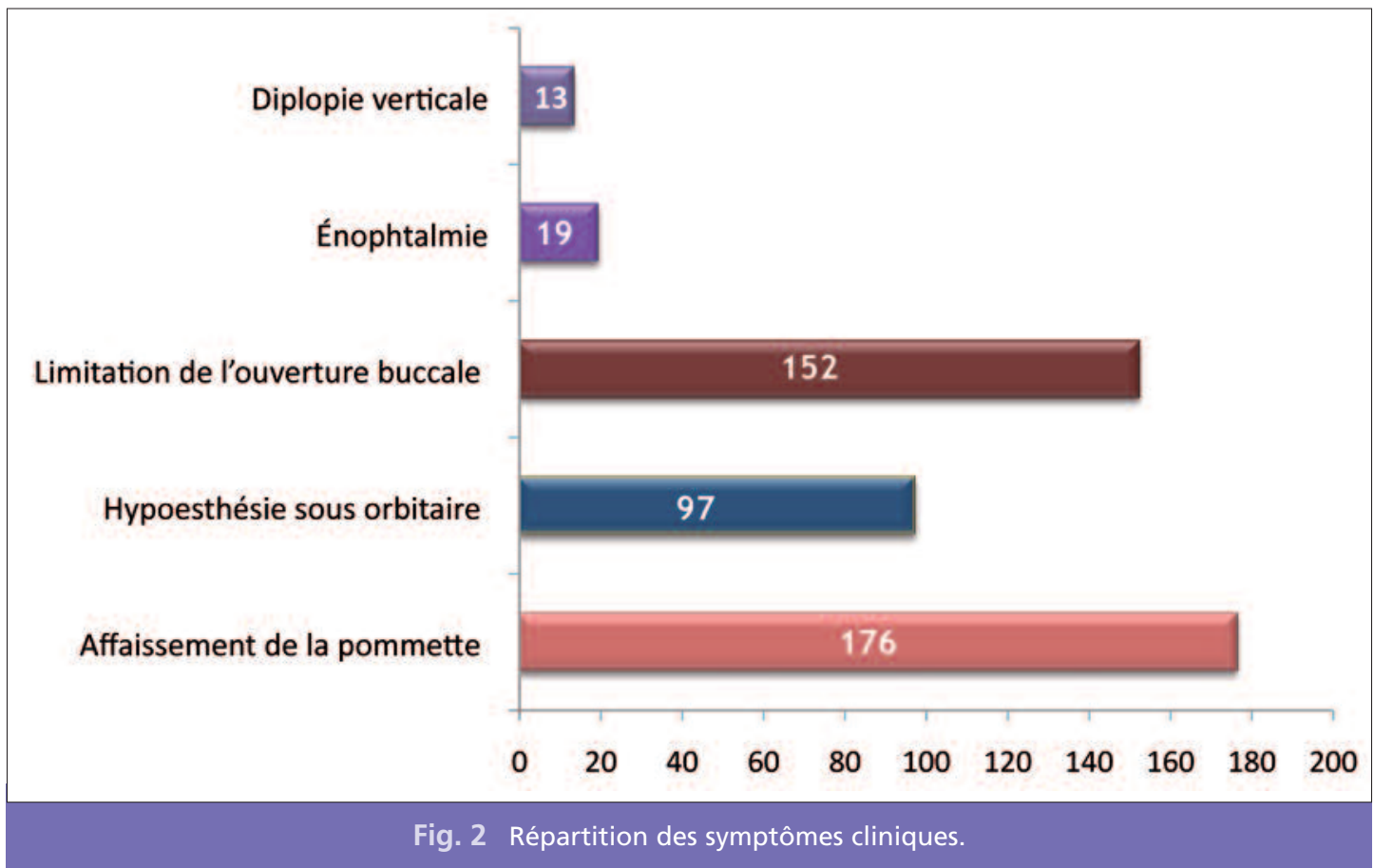

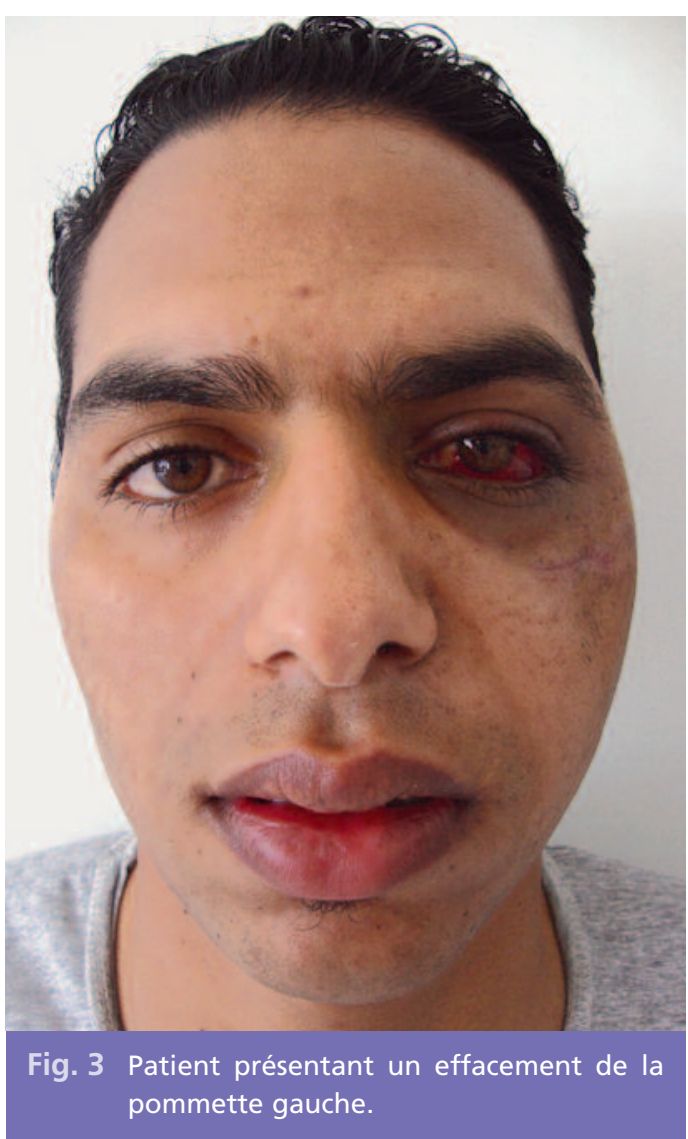

6,9\% des cas, 6 d'entre eux ont présenté une diplopie.

Les troubles fonctionnels (fig. 2) ont été les moins fréquents : la limitation de l'ouverture buccale (152 cas soit 55,1\%), I'hypoesthésie sous orbitaire (97 cas, 35,1\%). Treize patients ont rapporté une diplopie verticale objectivée systématiquement par le test de Lancaster. Cependant, deux patients n'ont pas pu bénéficier de ce test par défaut de moyens.

Le bilan radiologique a permis de poser le diagnostic et de réaliser une topographie lésionnelle précise (tableau 1).

Dans notre série, les indications opératoires ont dépendu du type de fracture (tableau 2). Le plancher orbitaire a nécessité une reconstruction dans 78 cas sur les 145 explorés, soit $53 \%$. La réduction a été contrôlée en post-opératoire immédiat par l'incidence de Blondeau, jugée satisfaisante dans $95 \%$ des cas. Aucun déplacement secondaire $n$ 'a été noté. 
Tableau I Répartition des traits de fractures.

\begin{tabular}{|c|c|}
\hline Catégorie & Fréquence \\
\hline A1 & 45 \\
A2 & 7 \\
A3 & 6 \\
B & 192 \\
C & 23 \\
D & 3 \\
\hline
\end{tabular}

Les séquelles immédiates (tableau 3) les plus fréquentes ont été représentées par les troubles de sensibilité $(19,2 \%)$, la diplopie $(1,8 \%)$, l'énophtalmie $(2,2 \%)$ et les défauts de projection $(4,7 \%)$.

Après un recul minimal de 12 mois, tous les malades qui ont gardé une énophtalmie ont été repris. Ce deuxième temps opératoire a été un échec, puisqu'aucune vraie amélioration n'a été constatée.

\begin{tabular}{|r|c|c|c|}
\hline \multicolumn{5}{|c|}{ Tableau II Indications opératoires. } \\
\hline Type de la fracture & Réduction fermée & Réduction ouverte & Abstention \\
\hline Catégorie A : & 39 & 3 & 3 \\
A1 & 0 & 5 & 2 \\
A2 & 0 & 5 & 1 \\
\hline Catégorie B & 49 & 134 & 9 \\
\hline Catégorie C & 0 & 23 & 0 \\
\hline Catégorie D & 0 & 3 & 0 \\
\hline Total & 88 & 173 & 15 \\
\hline
\end{tabular}

\begin{tabular}{|c|c|c|c|c|}
\hline \multicolumn{5}{|c|}{ Nombre de cas } \\
\hline & Suites immédiates & 3 mois & 6 mois & 1 an \\
\hline Hypoesthésie sous orbitaire & 53 & 31 & 19 & 3 \\
\hline Névralgies & 0 & 7 & 3 & 2 \\
\hline Diplopie & 5 & 2 & 1 & 1 \\
\hline Enophtalmie & 6 & 6 & 6 & 6 \\
\hline Défauts de projection & 13 & 13 & 13 & 13 \\
\hline Total & 77 & 59 & 42 & 25 \\
\hline
\end{tabular}

\section{Discussion}

Une bonne prise en charge clinique et radiologique des fractures de l'os zygomatique per- met de mieux retenir l'indication thérapeutique. Notre expérience valide certaines indica- 
tions pour la réduction orthopédique et souligne surtout l'intérêt de la réduction sanglante.

Sur le plan épidémiologique, la prédominance masculine $(89,5 \%)$ et l'âge jeune (32 ans) des patients s'expliquent par l'activité physique des malades, ce qui est compatible avec les études déjà publiées [2, 3].

On a constaté que les accidents de la voie publique constituent la cause la plus fréquente et représentent $64 \%$ de l'ensemble des étiologies, ce qui concorde avec les travaux de Tanaka [2] et Bouguila [4]. Pour Tadj et Kimble [5], les agressions physiques constituent la cause la plus fréquente dans les fractures de l'os zygomatique.

Sur le plan clinique, les signes morphologiques sont dominés par l'effacement de la pommette (fig. 3), signe qui doit faire évoquer une fracture du zygoma.

L'énophtalmie retrouvée chez $6,9 \%$ des cas peut s'expliquer par divers mécanismes, elle peut être immédiate ou apparaître secondairement, faisant suite à une exophtalmie par hématome et œdème orbitaire [6].

Les signes fonctionnels sont dominés par la limitation de l'ouverture buccale qui résulte soit d'un réflexe douloureux soit d'un conflit coronoïdo-malaire, Ce signe, présent dans $55,1 \%$ des cas, doit être chiffré pour suivre l'évolution.

L'hypoesthésie sous-orbitaire par compression ou attrition du nerf sous-orbitaire doit être recherchée avant toute attitude thérapeutique, car elle a un intérêt médico-légal. La plupart des troubles esthésiques régressent, sauf en cas de section du tronc nerveux. Dans notre série, ces troubles ont été constatés dans $35,1 \%$ des cas. Selon la littérature, l'incidence des déficits neurosensoriels dans les fractures de l'os zygomatique est de 10 à $50 \%$ [7]. Nos données concordent avec les chiffres fournis par Bouguila [4].

La diplopie est souvent méconnue et n'est décelée que quelques jours après le traumatisme du fait de l'œdème palpébral qui empêche la vision binoculaire (fig. 4). C'est dire l'importance du test de duction forcée en matière de diagnostic positif de la diplopie. II s'agit d'un déséquilibre oculomoteur objectivé cliniquement par l'étude de l'excursion oculaire. C'est un signe majeur des fractures du plancher orbitaire qui doit être recherché avant toute attitude thérapeutique, car il a un intérêt médico-légal. Dans notre série, la diplopie a été objectivée chez 13 patients soit 4,7\% des cas. Ces chiffres concordent avec ceux de la littérature [4]. Le test de Lancaster permet de quantifier la diplopie et d'en suivre l'évolution.

Les données topographiques des traits de fracture sont évaluées par l'imagerie. La radio en

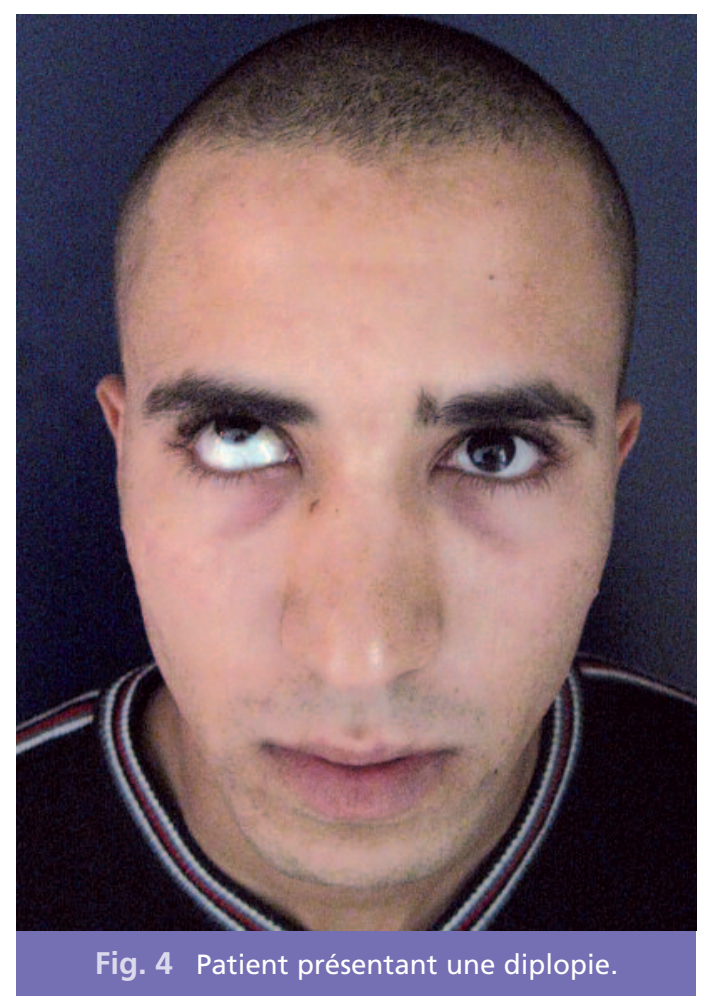


incidence de Blondeau est l'examen standard le plus demandé devant tout traumatisme latéro-facial. Un résultat négatif pourrait autoriser I'arrêt des investigations. Cependant, lorsque la clinique est évocatrice, la TDM avec des coupes coronales est indispensable, elle permet d'obtenir une topographie lésionnelle précise, de mettre en évidence une incarcération du contenu orbitaire et d'être un document iconographique pré-opératoire, voire médico-légal.

Les résultats obtenus, dans notre série, ont montré une nette prédominance des fractures disjonction de l'os zygomatique (fig. 5), ces résultats corroborent certaines études récentes
$[4,5]$. Le tableau 4 montre la grande variabilité de la répartition topographique des traits de fracture selon les pays et les études.

Le but du traitement est la restauration de la forme et de l'harmonie du visage et de rétablir I'orthophorie en position primaire avec la plus faible morbidité.

La réduction orthopédique par crochet de Ginestet nécessite un contrôle de la force appliquée lors de l'élévation. La force doit être bien contrôlée pour éviter un déplacement de la fracture dans la direction opposée et compromettre toute possibilité d'engrainement des fragments s'ils sont trop déplacés. Bien que le craquement qui se fait entendre au moment

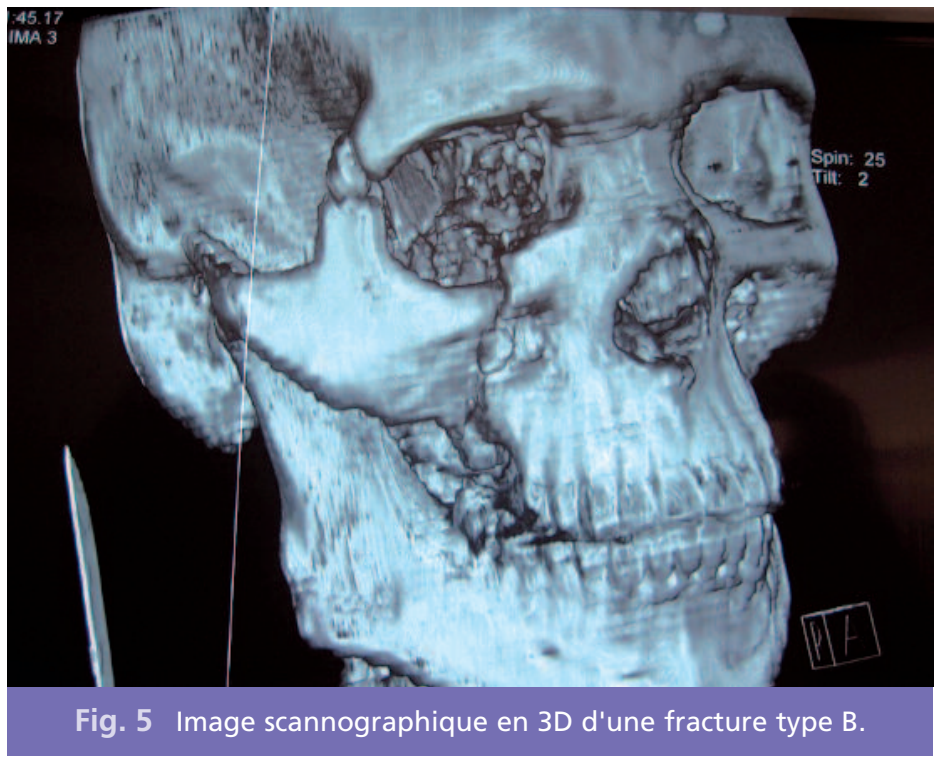

Tableau IV La topographie des traits de fractures selon les études.

\begin{tabular}{|c|c|c|c|c|}
\hline Type de fracture & Notre série & Bouguila et al. [4] & Zingg et al. [10] & Tadj et al. [5] \\
\hline A1 & 16,3 & 34,5 & 8 & 7,7 \\
\hline A2 & 2,5 & 1,4 & - & 6,3 \\
\hline A3 & 2,2 & 1,4 & - & 10,4 \\
\hline B & 69,6 & 43,7 & 5 & 60,6 \\
\hline C & 8,3 & 20 & 57 & 14,9 \\
\hline D & 1,1 & - & 33 & - \\
\hline
\end{tabular}


de la réduction indique que la réduction est complète, elle doit être confirmée cliniquement, notant que cette technique nécessite un chirurgien expérimenté [8].

La réduction sanglante nécessite des voies d'abord variables en fonction de la topographie des traits de fracture. La margelle infraorbitaire et le plancher de l'orbite, dans notre série, ont été abordés à travers une incision sous-tarsale, ce qui rejoint les travaux récents de Timóteo [9], incision qui offre un accès facile avec une morbidité minimale et une cicatrice esthétiquement acceptable. La voie sous-ciliaire fournit aussi un abord rapide et une approche directe mais le risque d'ectropion est élevé. Toutefois, I'incision transconjonctivale permet un excellent résultat esthétique mais l'accès est limité et elle n'est applicable qu'aux fractures isolées du plancher. L'incision coronale offre un excellent accès à l'arcade zygomatique et au complexe zygomatique, contribuant à une bonne réduction anatomique. Elle a aussi des inconvénients comme la longue durée du temps opératoire, le risque de lésion du nerf facial, les cicatrices disgracieuses chez les patients présentant une calvitie et une paresthésie du site exploité. Par conséquent, I'incision doit être judicieusement utilisée et non surexploitée et les indications strictement appliquées [10].

La jonction fronto-zygomatique est abordée par une incision latérale au niveau de la queue du sourcil ou par une voie palpébrale supérieure [11]. Pour le cintre maxillo-zygomatique, la voie vestibulaire supérieure reste la seule voie d'abord.

La contention des foyers de fracture est réalisée par des mini-plaques vissées, ce qui permet une reconstruction rapide anatomique et fonctionnelle [12].
L'utilisation d'une plaque d'ostéosynthèse au niveau de la jonction fronto-zygomatique et une autre au niveau de la margelle infra-orbitaire sont suffisantes pour l'obtention d'une symétrie anatomiquement correcte du zygoma. II n'existe aucune différence clinique entre des fixations en 2 et 3 points, comme le confirme également Kovács [12], à condition de faire le contrôle et le repositionnement du cintre maxillo-malaire.

Quant à I'utilisation de la fixation par fil d'acier, elle garde une place dans les fractures comminutives.

Pour les fractures isolées de l'arcade zygomatique $\mathrm{A} 1$, la réduction orthopédique est généralement suffisante. Les fractures du rebord orbitaire latéral A2 et celles de la margelle infra-orbitaire $A 3$ sont en général stables et ne nécessitent une réduction chirurgicale qu'en cas de déplacement.

Pour les fractures-disjonctions B nettement déplacées et instables, tous les auteurs indiquent une réduction ouverte et une fixation par des mini-plaques. Toutefois, la gestion des fractures moins sévères reste débattue. Les écoles qui optent pour le traitement conservateur [13], à savoir la réduction fermée (technique de Gillies, par exemple), estiment que les résultats cliniques obtenus par cette technique relativement simple sont satisfaisants et que les désagréments causés par la voie ouverte sont anéantis. Le camp opposé estime que les séquelles post-réduction sont fréquentes et difficiles à gérer; par conséquent, presque toutes les fractures, quel que soit leur type, doivent être directement abordées et fixées $[14,15]$.

Pour notre part, la conduite la plus pratique serait de différencier deux types d'indications : 


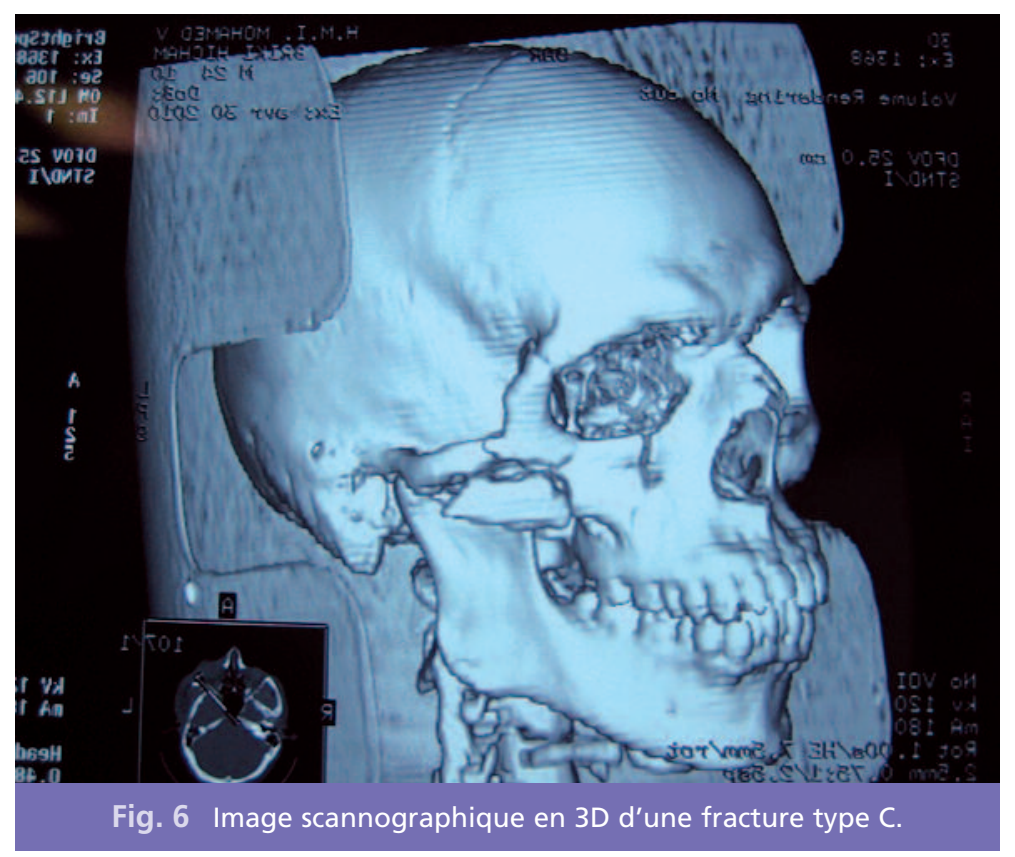

- une indication purement morphologique, où I'on réalise une réduction orthopédique et seuls les zygomas incorrectement réduits ou instables seront abordés ;

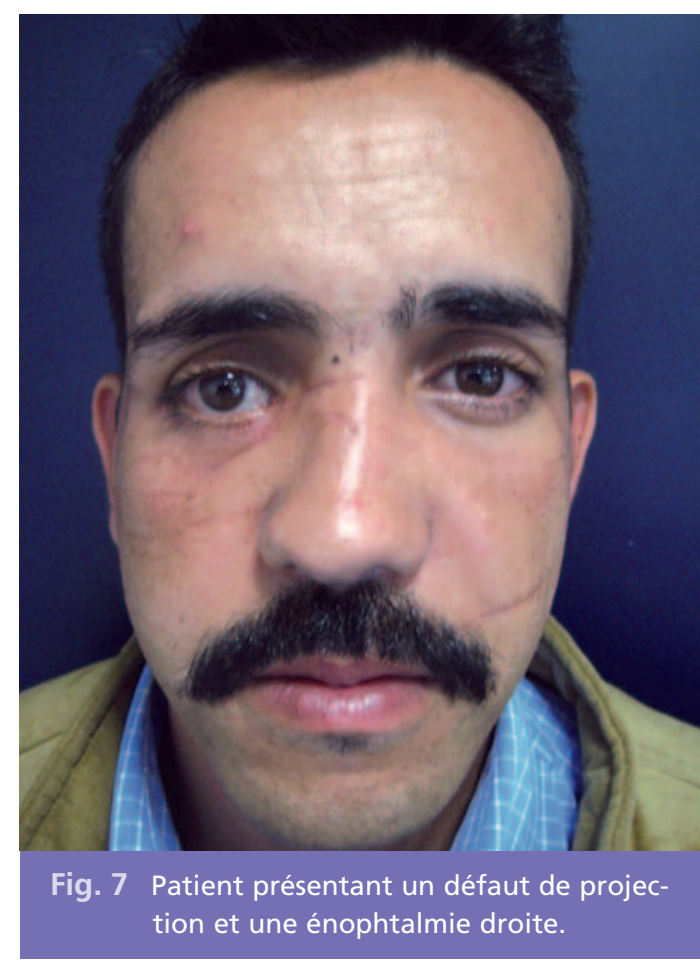

- une indication fonctionnelle, où la voie d'abord permet, en plus de la réduction, l'exploration du plancher, la désincarcération du contenu orbitaire, la réfection du plancher et le contrôle du nerf sous-orbitaire.

Pour les fractures de type $C$ (fig. 6) et $D$, tous les auteurs conviennent que celles-ci exigent une approche ouverte [4].

En ce qui concerne les séquelles, l'énophtalmie est le plus souvent le résultat d'un défaut de restauration du volume exact de l'orbite plutôt que la fibrose et la perte des tissus mous. La plupart des principes applicables à la gestion du traumatisme initial et leur respect devraient prévenir cette complication pour le plus grand nombre des patients [16].

Un traitement tardif ou incomplet des défauts de projection conduit le plus souvent à des préjudices esthétiques importants (fig. 7) [16]. La diplopie persistante pourrait s'expliquer par le retard de la prise en charge, source de fibrose [17]. Par ailleurs, l'atteinte nerveuse reste toujours de mise. 
La prise en charge des séquelles neurologiques reste un défi majeur, vu la fréquence de surve- nue et la persistance des troubles sensitifs dans le territoire infra-orbitaire [17].

\section{Bibliographie}

1. Zingg $M$, Chowdhury $K$, Lädrach $\mathrm{K}$, Vuillemin $\mathrm{T}$, Sutter F, Raveh J.

Treatment of 813 zygoma-lateral orbital complex fractures. New aspects.

Arch Otolaryngol Head Neck Surg 1991;117(6):611-20.

2. Tanaka N, Tomitsuka K, Shionoya $\mathrm{k}$, Andou $\mathrm{H}$, Kimijima $Y$, Tashiro $T$, Amagassa $\mathrm{T}$.

Aetiology

of maxillofacial fracture. Br J Oral Maxillofac Surg 1994;32(1):19-23.

3. al-Qurainy IA, Stassen LF, Dutton GN, Moos KF, el-Attar A.

The characteristics of midfacial fractures and the association with ocular injury: a prospectice study. Br J Oral Maxillofac Surg 1991;29(5):291-301.

4. Bouguila J, Zairi I, Khonsari RH, Hellali M, Mehri I, Landolsi A, et al. [Fractured zygoma : a review of 356 cases]. Ann Chir Plast Esthet 2008;53(6):495-503.

5. Tadj A, Kimble FW. Fractured zygomas. ANZ J Surg 2003;73(1-2):49-54.

6. Longaker MT, Kawamoto HK Jr. Evolving thoughts on correcting posttraumatic enophthalmos.
Plast Reconstr Surg 1998;101(4):899-906.

7. Vriens JP, Moos KF. Morbidity of the infraorbital nerve following orbitozygomatic complex fractures. J Craniomaxillofac Surg 1995;23(6):363-8.

8. Kaastad E, Freng A. Zygomatico-maxillary fractures. Late results after traction-hook réduction.

J Craniomaxillofac Surg 1989;17(5):210-4.

9. Timóteo CA, Chagas JF, Rapoport A, Denardin OV. Evaluation of palpebral subtarsal approach in surgical treatment of orbito-zigomatic fractures. Rev Col Bras Cir 2009;36(5):382-91.

10. Zhang QB, Dong YJ, Li ZB, Zhao JH.

Coronal incision for treating zygomatic complex fractures. J Craniomaxillofac Surg 2006;34(3):182-5.

11. Abouchadi A, Capon-Degardin N, Martinot-Duquennoy $\mathrm{V}$, Pellerin $\mathrm{P}$. Eyelid crease incision for lateral orbitotomy. Ann Chir Plast Esthet 2005;50(3):221-7.

12. Kovács $A F$, Ghahremani $M$. Minimization of zygomatic complex fracture treatment.
Int J Oral Maxillofac Surg 2001;30(5):380-3.

13. Carr RM, Mathog RH. Early and delayed repair of orbitozygomatic complex fractures.

J Oral Maxillofac Surg 1997;55(3):253-8.

14. O'Hara DE, DelVecchio DA, Bartlett SP, Whitaker LA. The role of microfixation in malar fractures: a quantitative biophysical study.

Plast Reconstr Surg 1996;97(2):345-50.

15. Czerwinski M, Martin M, Lee $C$.

Quantitative comparison of open reduction and internal fixation versus the Gillies method in the treatment of orbitozygomatic complex fractures.

Plast Reconstr Surg 2005;115(7):1848-54.

16. Becelli R, Carboni A, Cerulli G, Perugini M, lannetti G. Delayed and inadequalety treated malar fractures: evolution in the treatment, presentation of 77 cases, and review of the literature. Aesthetic Plast Surg 2002;26(2):134-8.

17. Folkestad L, Westin $\mathrm{T}$. Long-term sequelae after surgery for orbital floor fractures. Otolaryngol Head Neck Surg 1999;120(6):914-21. 


\section{SUMMARY}

\section{Fractured zygoma: about 276 cases}

\author{
Lahcen KHALFI \\ Abdeljalil ABOUCHADI \\ Jalal HAMAMA \\ Karim ELKHATIB \\ Mohamed NASSIH \\ Abdelkader RZIN
}

$$
\begin{aligned}
& \text { Keywords } \\
& \text { - zygomatic fracture } \\
& \text { - Morocco }
\end{aligned}
$$

Introduction: Zygomatic bone fractures have many anatomoclinic varieties at the origin of functional impact. The purpose of this work is to determine and to specify arising circumstances, clinical and radiological epidemiology, therapeutic attitude, and the future of these patients in average term.

Materials and methods: A retrospective study included patients with zygomatic bone fracture, addmitted at the facial surgery department of Mohammed $\mathrm{V}$ Military Instruction Hospital, Rabat; between March 1999 and April 2009.

Results: We included 276 files, the average age was 32 years old, with male predominance $(89,5 \%)$. Causes were dominated by the traffic public accidents $(64,2 \%)$. Diagnosis is especially clinic, confirmed by radiology. The open reduction was carried out in 173 cases, while $31,9 \%$ of cases necessitate a closed reduction; the most frequent sequels were sensory troubles $(19,2 \%)$.

Discussion: Pre-operative evaluation must include a complete ophtalmological evaluation. Surgical principles in zygoma fractures management are principally linked to the reeducation. This experience validates some indications for orthopedic reeducation, and underlines the interest of bloody reeducation with fixing by aimed mini veneers. Surgical indication depends on fracture type.

\section{NOUVELE REVUE}

\section{Droit et Médecine Bucco-Dentaire La revue de l'actualité en droit dentaire}

Directeur de la rédaction : Alain Béry

Rédacteurs en chef : Laurent Delprat et Charles Georget

Il y a plusieurs années, sous l'impulsion d'André Demichel, est apparue l'émergence du droit médical. Désormais, devant la spécialisation croissante des techniques et du droit, l'instauration d'un droit dentaire spécifique semble relever de l'évidence.

C'est dans cet esprit de reconnaissance que l'Association de Droit Dentaire, portée par l'indéfectible soutien de sa Présidente d'honneur Francine Demichel, a souhaité procéder à la création de cette nouvelle revue de "Droit et Médecine Bucco-Dentaire".

La publication, trimestrielle, permet de rendre compte rapidement de l'actualité juridique, qu'il s'agisse de nouvelles lois ou jurisprudences, réformes, études de cas, ou autres décisions touchant votre exercice quotidien.

Abonnez-vous en ligne!

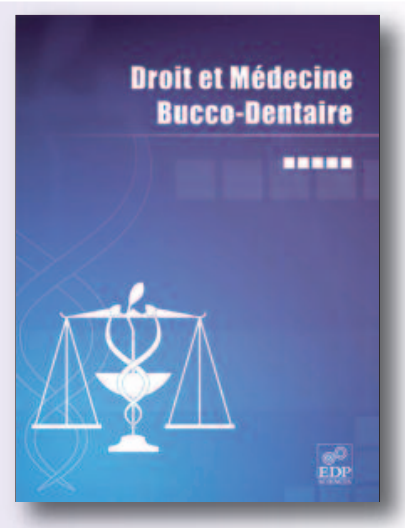

4 numéros / an

ISSN : 2116-1321 e-ISSN : $2116-133 \mathrm{X}$

\section{www.rdmbd.org}

